\title{
Interactive comment on "Improved parameterization of the weathering kinetics module in the PROFILE and ForSAFE models" by Harald Ulrik Sverdrup et al.
}

\section{Anonymous Referee \#1}

Received and published: 12 April 2020

This is not a scholarly publishable paper for a variety of reasons. The paper reads like a monograph - in fact, an old fashioned monograph where the author asserts his/her opinions. I do not find the paper to be publishable in this peer reviewed journal. Some examples of why I dont find this paper publishable are shown below.

1. The main reason to publish this paper might be because PROFILE as a model (and its descendents and modifications) has been amply used in the European system to assess impacts of acid rain. Nonetheless, the model is based on an approach that has never been fully or adequately discussed in the literature. In particular, kinetic constants in the model are often described only in Sverdrup, H.U., 1990. The Kinetics

Printer-friendly version

Discussion paper 
of Base Cation Release Due to Chemical Weathering. Lund University Press, Lund, Sweden, which is itself a monograph with many unsubstantiated assertions and questionable data sources. This paper does not address this limitation and only seems to make the problem worse. The authors should be encouraged to publish this paper as a monograph because this paper is impossible to peer review.

2. Apparently, one of the things that is new in this presentation is the addition of "braking functions". As far as I can tell, these are fudge factors that are not based on data nor theory. Some of the Al effect may be based on Oelkers' models but the Si function does not seem to even have that basis (Figure 9 shows no data). In fact, no reactive transport model can be used to simulate a natural field system accurately without fudge factors. Usually authors choose surface area as a fudge factor. As I see this paper, these authors are simply choosing braking factors as their fudge factors. I don't see why this is an advance. Especially when it is not adequately explained.

3. The abstract suggests that the paper treats $2 D$ and $3 D$ catchments but I do not see where that is described in the paper. What, in particular, is a 2D catchment? The assertions in the abstract are over reaching.

4. Most of the citations throughout are only to papers with Sverdrup as a co author. The many other papers that are cited are almost strictly cited as a footnote to one of the tables or they are briefly mentioned when reaction rate orders are chosen. But very little substantiation for choices is presented.

5. While the authors need to present specifics to build confidence in what they are doing, the paper almost entirely deals in generalities. For example, the first figure is a simple schematic of feedback loops. How does this help the paper? It has very little to contribute. Likewise, other figures are not helpful: for example, Figure 2 is ostensibly a comparison of field to model rates, but so little information is given that no interpretation of the figure can be made by the reader.

Printer-friendly version

Discussion paper

6. The first paragraph in Section 2. Methodology provides little in the way of useful 
content.

7. One of the interesting points of this paper and the model is that Sverdrup et al. point out that modelling feldspar dissolution as if the feldspar precipitates is inaccurate. . . and this is how all the other models treat the system. As written in the paper, however, the authors simply make an assertion that re-precipitation of feldspar cannot happen and so the TST treatment is not appropriate. Interestingly, new data seem to substantiate the authors' assertion. . .but are not mentioned or cited. The authors should see Zhu et al. 2020 in GCA. This is another good example of how the authors are not really pinned to data or to the literature at all. This could become a big paradigm shift, if all models shifted how they treat feldspar dissolution (as well as other minerals). But this is not adequately defended nor substantiated.

8. The authors assert that the way the model treats surface area has been reviewed in detail. Nonetheless, my understanding of what the model does with surf area is that it assumes a particle size distribution that then sets the surface area. This is perhaps not as robust or theoretically defensible as the authors assert or imply.

9. Some descriptions are impossible to understand: line 230 , "if some reactions occupy the same active mineral surface sites, the expression given above would change to a quadratic sum." I could give many other examples of sentences that were impossible for me to understand.

10. The caption of figure 6 says that the reaction pathways are shown according to Transition State Theory. . .which sounds good... but what does this mean? I don't see how this figure is related to TST and the caption does not make it clear. There are many examples like this where big words and big assertions are used or made that make what is being done here sound better than it really is. (Although who knows? It is all a mystery)

11. The authors do not explain partial causal loop diagrams and yet they present one. This is from systems theory but is not well explained here. It is slightly explained

Printer-friendly version

Discussion paper

3 
around lines 125 but the presentation is not adequate (if the reader is to understand the importance here).

12. I may have missed something but the authors seem to only include retardation of mineral reactions by organic molecules. Do they include acceleration by organic molecules (which is known to happen)?

13. The authors seem to think it is ok to define clay minerals to include non clays such as quartz. There are two definitions of clays...but the authors do not make this clear.

14. I did not understand the pathways in Table 2.

15. The authors cite a lot of experimental data papers in footnote 2. But they state that the data derive from these papers, but are not limited to these papers. This is not scholarly. How can we have faith in what they have done if we cannot figure out what they have done?

16. In the data table for kinetic constants, the authors write: " . . the regression of $\sim 20$ have yet to be published. In due time, these will get their own proper publications, it is beyond the scope of this study to do them in detail. Data and records from unpublished experiments and experiment evaluations by Sverdrup and Holmqvist are available on paper records held in a large number of binders at the Inland University of Applied Sciences, at Hamar, Norway. These data are no longer available in digital form due to computer system changes and data filing format changes that have occurred during the last 20 years. This documentation could be available in 1-2 years time, provided that funding for the redigitalization work can be obtained." I have never seen anything published with that sort of apology. I think it is inappropriate. If we cannot see the data, then the authors are simply making an assertion. Which minerals fall into this category?

17. Why don't these authors use standard units? See Figure $12: \mathrm{kmol} / \mathrm{m} 3$. Are these standard units in some field? If so, fine. If not, please use standard units.

Printer-friendly version

Discussion paper 
18. I could not figure out figures 13 nor 14 ...how did the authors parameterize the effect of $\mathrm{Al}$ and BC based on what I can see in the figures?

19. In some cases, I saw that the authors cited many more recent papers but I was left to wonder if they actually used those papers. Or were they just citing them to make it look like their compilation of kinetic constants is up to date? A few examples. On line 690 or so, they cite papers for the rate order with respect to CO2. Earlier, they cited a paper by Navarre-Sitchler and Thyne, but it is not cited here. Was that paper used here or not? Somehow, it seems like magic, they come up with a rate order of 0.6. It is unclear how they arrived at this value. Another mystery: "Estimates for some of the rate coefficients in Table 3 were based on mineral crystal structure analogies (citations)." Which minerals? And how did this analogy argument work? Likewise, I do not understand what the authors mean by Figure 22. "Data points drawn in". I am left with little confidence in the rate constants that the authors chose here.

20. Even when the authors seemed up to date in their citations, it was not clear that they actually used the new data to do anything. They cite a thesis and a paper about olivine reactions by A Olsen. Did they use those two references? What about the compilation by Palandri and Kharaka? How was that compilation used? $\mathrm{P}$ and $\mathrm{K}$ are cited, but was the compilation used? For example, in the specific discussion late in this paper about olivine reaction rate order, the Olsen and Rimstidt paper and the Olsen thesis were not cited. In fact, many many people have discussed the reaction rate orders for different minerals, but these discussions in the literature do not seem to have been considered here. Rather, it seems like ad hoc decisions have been made.

Interactive comment on Biogeosciences Discuss., https://doi.org/10.5194/bg-2019-464, 2020. 